University of Wollongong

Research Online

Faculty of Engineering - Papers (Archive)

Faculty of Engineering and Information

Sciences

$1-1-2009$

\title{
Synthesis of SmFeAsO by an easy and versatile route and its physical property characterization
}

\author{
V P. Awana \\ National Physical Laboratory, New Delhi \\ Anand Pal \\ National Physical Laboratory, New Delhi \\ Arpita Vajpayee \\ Natl Phys Lab, New Delhi 110012, India \\ H Kishan \\ Natl Phys Lab, New Delhi 110012, India \\ Gustavo A. Alvarez \\ gustavo@uow.edu.au
}

See next page for additional authors

Follow this and additional works at: https://ro.uow.edu.au/engpapers

Digipalrt of the Engineering Commons

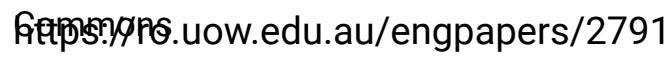

Network

Logo

Recommended Citation

Awana, V P.; Pal, Anand; Vajpayee, Arpita; Kishan, H; Alvarez, Gustavo A.; Yamaura, K; and TakayamaMuromachi, E: Synthesis of SmFeAsO by an easy and versatile route and its physical property characterization 2009, 07E316-1-07E316-3.

https://ro.uow.edu.au/engpapers/2791

Research Online is the open access institutional repository for the University of Wollongong. For further information contact the UOW Library: research-pubs@uow.edu.au 


\section{Authors}

V P. Awana, Anand Pal, Arpita Vajpayee, H Kishan, Gustavo A. Alvarez, K Yamaura, and E TakayamaMuromachi 


\section{AIP hapilie Phisis}

\section{Synthesis of SmFeAsO by an easy and versatile route and its physical property characterization}

V. P. S. Awana, Anand Pal, Arpita Vajpayee, H. Kishan, G. A. Alvarez et al.

Citation: J. Appl. Phys. 105, $07 E 316$ (2009); doi: 10.1063/1.3072382

View online: http://dx.doi.org/10.1063/1.3072382

View Table of Contents: http://jap.aip.org/resource/1/JAPIAU/v105/i7

Published by the American Institute of Physics.

\section{Related Articles}

Theoretical study of point-contact Andreev reflection spectroscopy for ferromagnetic-metal/multi-band superconductor junctions

J. Appl. Phys. 111, 07C518 (2012)

Pressure induced structural transition and enhancement of superconductivity in Co doped CeFeAsO Appl. Phys. Lett. 98, 012511 (2011)

Homologous series of iron pnictide oxide superconductors (Fe2As2)[Can+1(Sc,Ti)nOy] $(n=3,4,5)$ with extremely thick blocking layers

Appl. Phys. Lett. 97, 072506 (2010)

Intergrain connectivity of MgB2 ceramics studied by impedance analysis J. Appl. Phys. 108, 023901 (2010)

Calorimetric method of ac loss measurement in a rotating magnetic field Rev. Sci. Instrum. 81, 074702 (2010)

\section{Additional information on J. Appl. Phys.}

Journal Homepage: http://jap.aip.org/

Journal Information: http://jap.aip.org/about/about_the_journal

Top downloads: http://jap.aip.org/features/most_downloaded

Information for Authors: http://jap.aip.org/authors

\section{ADVERTISEMENT}

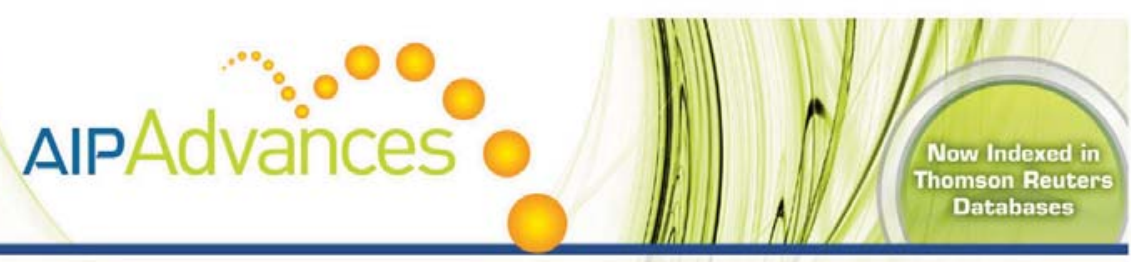

\section{Explore AIP’s open access journal: •Rapid publication \\ - Article-level metrics \\ Post-publication rating and commenting}




\title{
Synthesis of SmFeAsO by an easy and versatile route and its physical property characterization
}

\author{
V. P. S. Awana, ${ }^{1, a)}$ Anand Pal, ${ }^{1}$ Arpita Vajpayee, ${ }^{1}$ H. Kishan, ${ }^{1}$ G. A. Alvarez, ${ }^{2}$ \\ K. Yamaura, ${ }^{3}$ and E. Takayama-Muromachi ${ }^{3}$ \\ ${ }^{1}$ National Physical Laboratory, Dr. K.S. Krishnan Marg, New Delhi 110012, India \\ ${ }^{2}$ Institute for Superconducting and Electronic Materials, University of Wollongong, New South Wales 2522, \\ Australia \\ ${ }^{3}$ Advanced Nano-Materials Laboratory, National Institute for Material Science (NIMS), Tsukuba, \\ Ibaraki 305-0044, Japan
}

(Presented 11 November 2008; received 16 September 2008; accepted 20 November 2008; published online 9 March 2009)

\begin{abstract}
We report synthesis, structure, electrical transport, and heat capacity of SmFeAsO. The title compound is synthesized by one-step encapsulation of stoichiometric FeAs, $\mathrm{Sm}$, and $\mathrm{Sm}_{2} \mathrm{O}_{3}$ in an evacuated $\left(10^{-5}\right.$ Torr) quartz tube by prolong $(72 \mathrm{~h})$ annealing at $1100{ }^{\circ} \mathrm{C}$. The as-synthesized compound is crystallized in tetragonal structure with $P 4 / \mathrm{nmm}$ space group having lattice parameters $a=3.93726(33)$ A and $c=8.49802(07)$ A. The resistance $(R-T)$ measurements on the compound exhibited ground state spin-density-wave (SDW)-like metallic steps below $140 \mathrm{~K}$. Heat capacity $C_{P}(T)$ measurements on the title compound, showed an anomaly at around $140 \mathrm{~K}$, which is reminiscent of the SDW ordering of the compound. At lower temperatures the $C_{P}(T)$ shows a clear peak at around $4.5 \mathrm{~K}$. At lower temperature below $20 \mathrm{~K}, C_{p}(T)$ is also measured under an applied field of $7 \mathrm{~T}$. It is concluded that the $C_{P}(T)$ peak at $4.5 \mathrm{~K}$ is due to the antiferromagnetic ordering of $\mathrm{Sm}^{3+}$ spins. These results are in confirmation with ordering of $\mathrm{Sm}$ in $\mathrm{Sm}_{2-x} \mathrm{Ce}_{x} \mathrm{CuO}_{4}$. (C) 2009 American Institute of Physics. [DOI: 10.1063/1.3072382]
\end{abstract}

\section{INTRODUCTION}

Very recent invention of superconductivity of up to $58 \mathrm{~K}$ in $\mathrm{REFeAsO}(\mathrm{RE}=\mathrm{La}, \mathrm{Pr}, \mathrm{Sm}, \mathrm{Nd}, \mathrm{Gd})$ has been of tremendous interest to the scientific community. ${ }^{1-10}$ Besides the $\mathrm{Cu}$ based high $T_{c}$ superconductors (HTScs), ${ }^{11}$ the REFeAsO is the only known class of superconductors until date, having their superconducting transition temperature $\left(T_{c}\right)$ outside the so-called strong BCS (Bardeen Cooper and Schreifer) limit, i.e., $40 \mathrm{~K}$. Further, the Fe based compound provides an excellent opportunity to the theoreticians to think out side the cuprate families (HTSc) in search for the mechanism of high $T_{c}$ superconductivity. ${ }^{12-15}$ Unlike the HTSc, the synthesis route for the $\mathrm{REFeAsO}$ superconductors is quite complicated. Basically this is the reason that even after 4-6 months of their invention the gold rush is not like HTSc. In late 1986 and early 1987-1988, the reporting of $T_{c}$ was on daily basis like newspaper updates. Although the cond-mat arxiv preprint cite brings about five articles on daily basis in last 2-3 months, the material is yet confined mainly to couple of Japanese, Chinese, and some USA and Europe laboratories. In fact, the REFeAsO class of compounds is mainly synthesized by the high-pressure high-temperature (HPHT) process with pressure as high as $4-6 \mathrm{GPa}$ at $1250{ }^{\circ} \mathrm{C} .{ }^{4-6}$ Many researchers do not have the HPHT synthesis technique available in their laboratories. Some reports are available for the normal pressure synthesis route as well. ${ }^{1-3,7-10}$ The key step for normal pressure synthesis is to encapsulate the sample in

a) Author to whom correspondence should be addressed. FAX: 0091-11-45609310. Tel.: 0091-11-45608329. Electronic mail: awana@mail.nplindia.ernet.in. URL: www.freewebs.com/vpsawana/.
evacuated/Ar filled quartz tube and annealing up to $1150{ }^{\circ} \mathrm{C}$. One of the difficulties in this process is the stability of quartz at high temperature $\left(1150{ }^{\circ} \mathrm{C}\right)$. In particular, when carriers are doped via fluorine (F) doping, the quartz tube corrodes and resulting breaking of vacuum. In the current communication, we report synthesis of $\mathrm{SmFeAsO}$ in an evacuated $\left(10^{-5}\right.$ Torr $)$ quartz tube by prolong $(72 \mathrm{~h})$ annealing at

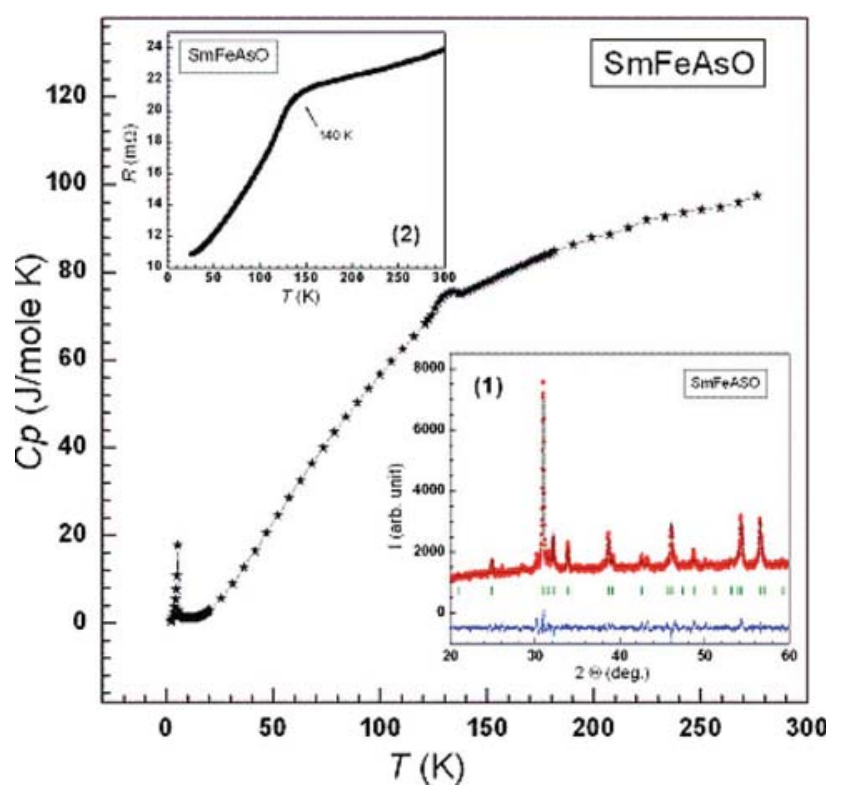

FIG. 1. (Color online) Specific heat of SmFeAsO sample (main panel). Observed and fitted XRD pattern (inset 1) and resistivity vs temperature plot (inset 2). 
TABLE I. Rietveld refined structure parameters of SmFeAsO. Space group: $P 4 / \mathrm{nmm}\left[a=3.93726(33) \mathrm{A}\right.$ and $c=8.49802(07)$ A $R_{p}: 3.26 \%, R_{\mathrm{wp}}$ : $\left.4.20 \%, R_{\text {exp }}: 2.47 \%, \chi^{2}: 2.90\right]$.

\begin{tabular}{lccclr}
\hline \hline Atom & Site & $x$ & $y$ & \multicolumn{1}{c}{$z$} & Occupancy \\
\hline $\mathrm{Sm}$ & $2 c$ & 0.25 & 0.25 & $0.1356(8)$ & $92.5(9)$ \\
$\mathrm{Fe}$ & $2 b$ & 0.75 & 0.25 & 0.5 & $100.1(4)$ \\
$\mathrm{As}$ & $2 c$ & 0.25 & 0.25 & $0.6514(15)$ & $94.3(8)$ \\
$\mathrm{O}$ & $2 a$ & 0.75 & 0.25 & 0 & $95.3(6)$ \\
\hline \hline
\end{tabular}

$1100{ }^{\circ} \mathrm{C}$. The resultant compounds are found to be nearly single phase but not superconducting due to lack of carriers. The resistivity and heat capacity measurements established the spin-density wave (SDW)-type ground state of the compound below $140 \mathrm{~K}$. Further, the $\mathrm{Sm}^{3+}$ spins order antiferromagnetic (AFM) below $4.5 \mathrm{~K}$ in this compound.

\section{EXPERIMENTAL}

Stoichiometric amounts of FeAs, $\mathrm{Sm}$, and $\mathrm{Sm}_{2} \mathrm{O}_{3}$ are weighed and mixed thoroughly in formula ratio $\mathrm{SmFeAsO}$. The weighed and mixed palletized powders are encapsulated in stainless steel tubes having small pinholes at both the ends. These are further encapsulated in an evacuated $\left(10^{-5}\right.$ Torr) quartz tube and annealed at $1100{ }^{\circ} \mathrm{C}$ for over 72
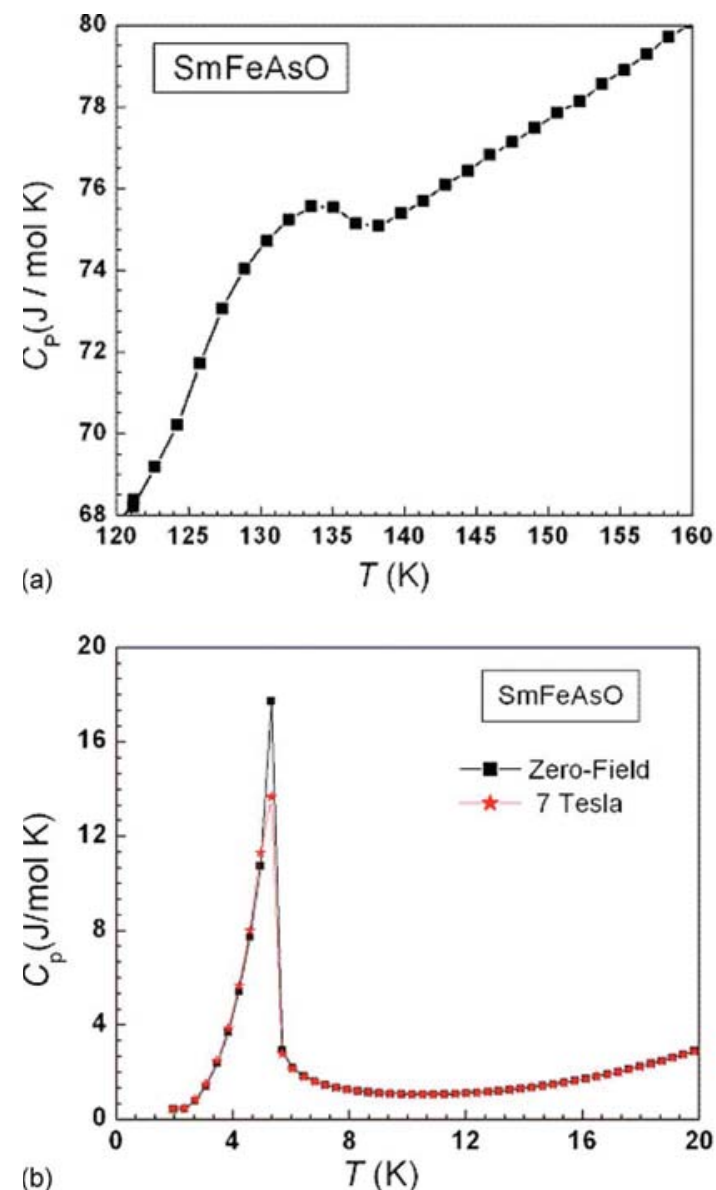

$\mathrm{h}$, respectively, in a single step, followed by cooling to room temperature over a span of $12 \mathrm{~h}$. This procedure is more or less similar to that as reported earlier. ${ }^{1,3,16}$ The x-ray diffraction pattern of the compound was taken on Rigaku mini-flex II diffractometer. The resistivity measurement was carried out by conventional four-probe method on a close cycle refrigerator in temperature range of 12-300 K. Heat capacity measurements were carried out on Quantum design Physical Property Measurement System.

\section{RESULTS AND DISCUSSION}

The room temperature powdered sample's x-ray diffraction (XRD) pattern and its Rietveld analysis are shown in lower inset of Fig. 1. The XRD pattern of the compound is fitted on the basis of tetragonal, $P 4 / \mathrm{nmm}$ space group. It indicates that besides the majority phase (tetragonal $P 4 / \mathrm{nmm}$ ) few weak impurity lines either from Fe, As, FeAs, or SmAs are also seen in the XRD pattern. The refined structural parameters of the studied sample are listed in Table I. The lattice parameters are found to be $a=3.93726(33)$ A and $c=8.49802(07)$ A. These values of lattice parameters are in confirmation with earlier reports. ${ }^{3,17-19}$ The lower inset of Fig. 1 shows the reasonably good fitting of the observed pattern of XRD.
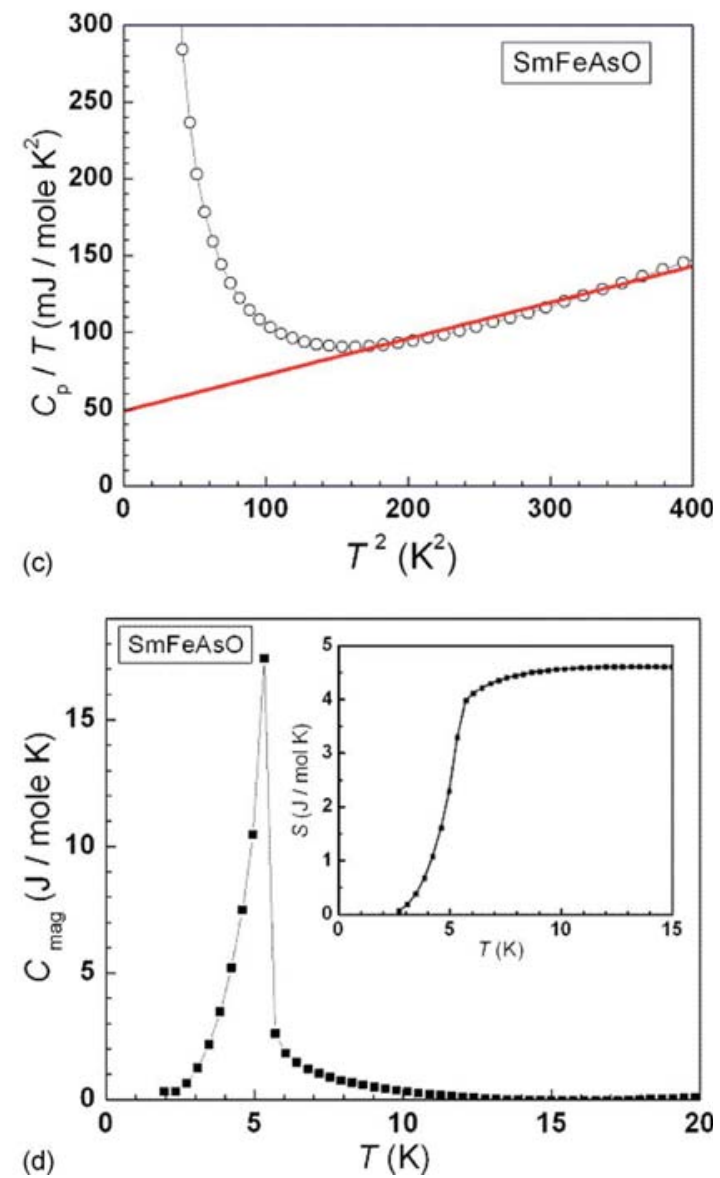

FIG. 2. (Color online) (a) Enlarged view of specific heat of SmFeAsO in the high-temperature range. (b) Enlarged view of specific heat of SmFeAsO in the low temperature range at $H=0$ and $7 \mathrm{~T}$. (c) $C_{p} / T$ vs $T^{2}$ plot for SmFeASO in $H=0 \mathrm{~T}$ and its linear fitting. (d) Magnetic specific heat of SmFeAsO and entropy associated with the magnetic transition (in inset) 
Resistance versus temperature $(R-T)$ plot of the SmAs$\mathrm{FeO}$ compound is shown in upper inset of Fig. 1. The $R-T$ behavior is less metallic comparatively with a relatively high resistance around $24 \mathrm{~m} \Omega$ at room temperature, which decreases slightly as the temperature goes down to around 140 $\mathrm{K}$. At $140 \mathrm{~K}$, the resistance drops abruptly. Below $140 \mathrm{~K}$ the $\mathrm{SmAsFeO}$ exhibits a metallic step down to the lowest measured temperature of $20 \mathrm{~K}$. The sharp metallic step below $140 \mathrm{~K}$ is in agreement with the earlier reported results ${ }^{1-10}$ and it arises due to the SDW magnetic structure of the compound.

Heat capacity $\left(C_{P}\right)$ versus temperature plot for the presently studied $\mathrm{SmAsFeO}$ compound is shown in main panel of Fig. 1. The absolute value of $C_{P}$ at $200 \mathrm{~K}$ is around $90 \mathrm{~J} / \mathrm{mol} \mathrm{K}$, which is although comparable but slightly less than the only other available report on $C_{P}(T)$ of $\mathrm{SmAsFeO}$ compound. ${ }^{20}$ With decrease in $T$ the $C_{P}$ goes down continuously, in a similar fashion as in Ref. 20. A distinct hump/kink is seen in $C_{P}(T)$ at around $140 \mathrm{~K}$. Interestingly this is the same temperature, where the metallic step is observed in $R(T)$. The metallic step is reminiscent of the SDW magnetic anomaly in the system. It is also known that besides SDW the ground state nonsuperconducting REFeAsO systems go through a structural phase transition simultaneously. With further decrease in temperature the $C_{p}(T)$ goes down before exhibiting a sharp peak at around $4.5 \mathrm{~K}$. The sharp peak at around $4.5 \mathrm{~K}$ is seen earlier in Ref. 20 as well and is assigned to the AFM ordering of $\mathrm{Sm}^{3+}$ spins. In brief the $C_{p}(T)$ measurements exhibited a shallow hump at around $140 \mathrm{~K}$ and a sharp peak at $4.5 \mathrm{~K}$. The former is probably due to the SDW magnetic ordering and the structural phase transition and the later from AFM ordering of $\mathrm{Sm}^{3+}$ spins. To understand the nature of the observed two characteristic $C_{P}(T)$ peaks i.e., $C_{P}(140 \mathrm{~K})$ and $C_{P}(4.5 \mathrm{~K})$, the same are zoomed and shown in Figs. 2(a) and 2(b), respectively. It is noted that sharp specific heat peak obtained at $4.5 \mathrm{~K}$ at zero magnetic field does shift anywhere on temperature scale in the application of $7 \mathrm{~T}$ magnetic field.

We have separated the magnetic and nonmagnetic contributions to $C_{p}$ by fitting of data in temperature range $12 \mathrm{~K} \leq T \leq 20 \mathrm{~K}$ using the equation ${ }^{2021}$

$$
C_{p}{ }^{\mathrm{NM}}(T)=\gamma T+\beta T^{3},
$$

where the linear term corresponds to the electronic contribution and cubic term corresponds to lattice contribution to the specific heat. In Fig. 2(c), $C_{p} / T$ versus $T^{2}$ is plotted for SmFeASO in zero magnetic field together with the fitting values (solid line). The values of $\gamma$ and $\beta$ are found to be $48.79 \mathrm{~mJ} / \mathrm{mole}^{2}$ and $0.24 \mathrm{~mJ} / \mathrm{mole}^{4}$, respectively. This value of $\gamma$ is lower than that obtained by Ding et al. in Ref. 20 . The contribution of magnetic correlation to the measured $C_{P}(T)$ is calculated by using the equation ${ }^{2021}$

$$
C_{p}{ }^{\mathrm{mag}}(T)=C_{p}(T)-C_{p}^{\mathrm{NM}}(T)
$$

and is plotted in Fig. 2(d). The temperature dependence of entropy associated with the magnetic transition is calculated from the $C_{p}{ }^{\mathrm{mag}}(T)$ and shown in inset of Fig. 2(d). The magnetic entropy saturates at temperature above $4.5 \mathrm{~K}$ to a value $\approx 4.5 \mathrm{~J} / \mathrm{mole} \mathrm{K}$. The usual entropy value of a doublet ground state of $\mathrm{Sm}^{+3}$ is $1.85 R \ln 2$, where $R$ is the gas constant. The estimated entropy value is obviously lower than the expected one of a doublet ground state. The reduced entropy can be explained by the fact that itinerant electrons with heavy effective mass are involved in the transition ${ }^{21}$

In summary, the specific heat of nonsuperconducting $\mathrm{SmFeAsO}$ compound has been studied systematically. First, a jump in specific heat value is observed at $140 \mathrm{~K}$, which is attributed to structural or SDW transition. Again a sharp peak is obtained at $T=4.5 \mathrm{~K}$ corresponding to the AFM ordering of $\mathrm{Sm}^{+3}$ ions in the system.

${ }^{1}$ Y. Kamihara, T. Watanabe, M. Hirano, and H. Hosono, J. Am. Chem. Soc. 130, 3296 (2008).

${ }^{2}$ H. Takahashi, K. Igawa, K. Arii, Y. Kamihara, M. Hirano, and H. Hosono, Nature (London) 453, 376 (2008).

${ }^{3}$ X. H. Chen, T. Wu, G. Wu, R. H. Liu, H. Chen, and D. F. Fang, Nature (London) 453, 761 (2008).

${ }^{4}$ H. Kito, H. Eisaki, and A. Iyo, J. Phys. Soc. Jpn. 77, 063707 (2008).

${ }^{5}$ Z. A. Ren, J. Yang, W. Lu, W. Yi, G. C. Che, X. L. Dong, L. L. Sun, and Z. X. Zhao, Mater. Res. Innovations 12, 105 (2008).

${ }^{6}$ G. F. Chen, Z. Li, D. Wu, J. Dong, G. Li, W. Z. Hu, P. Zheng, J. L. Luo, and N. L. Wang, Phys. Rev. Lett. 100, 247002 (2008).

${ }^{7}$ F. Hunte, J. Jaroszynski, A. Gurevich, D. C. Larbalestier, R. Jin, A. S. Sefat, M. A. McGuire, B. C. Sales, D. K. Christen, and D. Mandrus, Nature (London) 453, 903 (2008).

${ }^{8}$ W. Lu, J. Yang, X. L. Dong, Z. A. Ren, G. C. Che, and Z. X. Zhao, N. J. Phys. 10, 063026 (2008).

${ }^{9}$ C. R. dela Cruz, Q. Huang, J. W. Lynn, J. Li, W. Ratcliff II, J. L. Zarestky, H. A. Mook, G. F. Chen, J. L. Luo, N. L. Wang, and P. Dai, Nature (London) 453, 899 (2008).

${ }^{10}$ A. S. Sefat, M. A. McGuire, B. C. Sales, R. Jin, J. Y. Howe, and D. Mandrus Phys. Rev. B 77, 174503 (2008).

${ }^{11}$ J.G. Bednorz and K.A. Muller, Z. Phys. B: Condens. Matter 64, 189 (1986).

${ }^{12}$ K. Haule, J. H. Shim, and G. Kotliar, Phys. Rev. Lett. 100, 226402 (2008).

${ }^{13}$ D. J. Singh and M. H. Du, Phys. Rev. Lett. 100, 237003 (2008).

${ }^{14}$ Z. P. Yin, S. Lebègue, M. J. Han, B. Neal, S. Y. Savrasov, and W. E. Pickett, Phys. Rev. Lett. 101, 047001 (2008).

${ }^{15}$ G. Baskaran, e-print arXiv:0804.1341v2.

${ }^{16}$ J. Dong, H. J. Zhang, G. Xu, Z. Li, G. Li, W. Z. Hu, D. Wu, G. F. Chen, X. Dai, J. L. Luo, Z. Fang, and N. L. Wang, Europhys. Lett. 83, 27006 (2008).

${ }^{17}$ V. G. Hadjiev, M. N. Iliev, K. Sasmal, Y. Y. Sun, and C. W. Chu, Phys. Rev. B 77, 220505 (2008) (R).

${ }^{18}$ A. Martinelli, M. Ferretti, P. Manfrinetti, A. Palenzona, M. Tropeano, M. R. Cimberle, C. Ferdeghini, R. Valle, M. Putti, and A. S. Siri, Supercond. Sci. Technol. 21, 095017 (2008).

${ }^{19}$ P. Quebe, L. J. Terbuchte, and W. Jeitschko, J. Alloys Compd. 302, 70 (2000).

${ }^{20}$ L. Ding, C. He, J. K. Dong, T. Wu, R. H. Liu, X. H. Chen, and S. Y. Li, Phys. Rev. B 77, 180510 (2008).

${ }^{21}$ B. K. Cho, J. H. Kim, and Y. J. Kim, B. H. O, J. S.Kim, and R. Stewart, Phys. Rev. B 63, 214504 (2001). 